\title{
The Expressions of Wnt/ $\beta$-catenin Pathway-Related Components in Brainstem Gliomas
}

\author{
Wenhao Wu, Yongji Tian, Hong Wan, Yongmei Song, Junhua Li, Liwei Zhang
}

\begin{abstract}
Background: The overall prognosis of brainstem gliomas is very poor, and the current treatment cannot significantly prolong the overall survival of these patients; therefore, studying the molecular biological mechanisms of the occurrence and development of brainstem gliomas has important significance for their treatment. The Wnt/ $\beta$-catenin signaling pathway is closely associated with the occurrence and development of tumors, but its relationship with brainstem gliomas remains unclear. Methods: This study used Western blot and immunohistochemistry methods to detect the expressions of Wnt/ $\beta$-catenin signaling pathway-related components such as Wnt-1, Wnt-2, $\beta$-catenin and C-myc in six cases of normal brain tissues and 24 cases of brainstem gliomas and analyzed the relationship between their expressions and clinicopathological characteristics. Results: Wnt-1 had no obvious expression in normal brain tissues and did not show any significant difference between high- and low-grade brainstem gliomas; the expressions of Wnt-2, $\beta$-catenin and C-myc in high-grade brainstem gliomas were significantly higher than that in low-grade brainstem gliomas and normal brain tissues and were positively correlated with the expression of Ki-67. Moreover, the expressions of Wnt-2 and C-myc were significantly associated with the prognosis of brainstem glioma patients; additionally, there was a trend toward increased $\beta$-catenin expression with shorter survival, but there was no statistical difference. Conclusions: Wnt/ $\beta$-catenin signaling pathway might be abnormally activated and plays an important role in the occurrence and development of brainstem gliomas. Wnt-2, $\beta$-catenin and C-myc may be potential targets for brainstem glioma treatment.
\end{abstract}

RÉSUMÉ: Expression de composantes reliées à la voie de signalisation Wnt/ß $\mathbf{\beta}$-caténine dans les gliomes du tronc cérébral. Contexte : Le pronostic général des gliomes du tronc cérébral est très mauvais et le traitement actuel ne prolonge pas significativement la survie de ces patients. Il est donc important d'étudier les mécanismes de biologie moléculaire liés à leur apparition et à leur développement afin d'en améliorer le traitement. La voie de signalisation Wnt/ß-caténine est étroitement associée à l'apparition et au développement de tumeurs. Cependant, on ignore sa relation aux gliomes du tronc cérébral. Méthode : Dans cette étude, nous avons utilisé le buvardage Western ainsi que des méthodes d'immunohistochimie pour détecter l'expression de composantes reliées à la voie de signalisation Wnt/ $\beta$-caténine, telles Wnt- 1 , Wnt- 2 , $\beta$-caténine et $\mathrm{C}$-myc, dans 6 cerveaux normaux et 24 gliomes du tronc cérébral et nous avons analysé la relation entre leur expression et les caractéristiques anatomo-cliniques des tumeurs. Résultats : Wnt-1 n'avait pas d'expression apparente dans les tissus cérébraux normaux et il n'y avait pas de différence significative entre les gliomes du tronc cérébral de haut grade et ceux de bas grade de malignité. L'expression de Wnt-2, de $\beta$-caténine et de C-myc dans les gliomes du tronc cérébral de haut grade était significativement plus élevée que dans ceux de bas grade et dans le tissu cérébral normal, et leur expression était corrélée de façon positive à l'expression de Ki-67. De plus, l'expression de Wnt-2 et de C-myc était associée de façon significative au pronostic des patients atteints de gliomes du tronc cérébral. Une expression augmentée de $\beta$-caténine avait tendance à être associée à une survie plus courte, mais la différence n'était pas significative au point de vue statistique. Conclusions : Il se peut que la voie de signalisation Wnt/ $\beta$-caténine soit activée de façon anormale et joue un rôle important dans l'apparition et le développement des gliomes du tronc cérébral. Wnt-2, $\beta$-caténine et C-myc pourraient constituer des cibles de traitement du gliome du tronc cérébral.

Can J Neurol Sci. 2013; 40: 355-360

The incidence of brainstem glioma accounts for $77.4 \%$ of brainstem tumors, making it one of the neurosurgical diseases with the greatest and most direct impact on life and neurological functions. Despite the progress of microneurosurgery, radiotherapy and chemotherapy, the overall prognosis of brainstem glioma patients has not been significantly improved. The median progression-free survival time of malignant brainstem glioma is five to six months, the median survival time is $9-12$ months, and the two-year survival rate is only $10-25 \% ; 1,2$ however, the underlying molecular biological mechanisms remain unclear. Because of the importance of anatomical structures and physiological functions of the brainstem, the surgical risk is relatively high, and the collection of brainstem glioma specimens has certain difficulties; thus, the basic study of brainstem gliomas and the improvement of clinical treatments are somewhat limited. The Beijing Tiantan Hospital, affiliated

From the Department of Neurosurgery (WW, YT, LZ), Beijing Tiantan Hospital; Department of Biochemistry (HW, JL), Beijing Neurosurgical Institute, Capital Medical University; State Key Laboratory of Molecular Oncology (YS), Cancer Institute and Hospital, Chinese Academy of Medical Sciences and Peking Union Medical College, Beijing, P.R. China.

Received September 13, 2012. Final Revisions Submitted November 15, 2012 Correspondence to: Liwei Zhang, Department of Neurosurgery, Beijing Tiantan Hospital, Capital Medical University, No. 6 Tiantan Xili, Dongcheng District, Beijing, 100050, P.R. China. Email: zhangliweittyy@yahoo.com.cn. 
with Capital Medical University, performs approximately 20 brainstem glioma surgeries each year. This provides opportunities to conduct molecular biological studies of brainstem gliomas.

The abnormal activation of $\mathrm{Wnt} / \beta$-catenin signaling pathway is closely associated with the occurrence and development of many human tumors. Recently, target therapies through interference or blocking of $\mathrm{Wnt} / \beta$-catenin signaling pathway to observe whether the tumor progression can be blocked or delayed have become a major focus of tumor research. ${ }^{3-5}$ However, the relationship between Wnt/ $/$-catenin signaling pathway and gliomas, especially brainstem gliomas, remains unclear. In this study, we use Western blot and immunohistochemistry methods to detect the expression of the components involved in Wnt/ $\beta$-catenin signaling pathway, such as Wnt-1, Wnt-2, $\beta$-catenin and the downstream target gene Cmyc, in brainstem gliomas to explore the relationship between their occurrence, development and prognosis and the Wnt/ $\beta$ catenin signaling pathway to provide a molecular basis for future target therapies of brainstem glioma.

\section{Materials And Methods}

\section{Tissue samples}

With Institutional Review Board approval, freshly resected brainstem glioma samples were obtained from Beijing Tiantan Hospital and then stored in liquid nitrogen prior to use. None of the patients had been subjected to radiotherapy and/or chemotherapy prior to surgery. According to the World Health Organization classification (WHO 2007), the tumors were pathologically classified as low grade (WHO I-II) or high grade (WHO III-IV). The histological subtypes and pathologic grades of all brainstem glioma samples, which were confirmed by two pathologists independently, are given in Table 1. A total of 24 cases of tumor samples, including 16 low-grade brainstem gliomas and eight high-grade gliomas, were collected for this study. There were 14 males and 10 females, varying in age from 3 to 64 years (average 27.3 years). The tumors were located at midbrain in six cases, at pons in nine cases, and medulla nine cases. Non-neoplastic brain tissues from six patients with intractable epilepsy were also included as controls. Clinical data of the patients were collected and follow-up of all the patients was performed. Before enrollment, each patient in this study signed a written informed consent.

\section{Western blot}

Frozen samples were ground in liquid nitrogen and were homogenized in lysis buffer [phosphate buffered saline (PBS), $50 \mathrm{mM}$ Tris, $150 \mathrm{mM} \mathrm{NaCl}, 1 \%$ nonidet P-40 (NP-40), $0.5 \%$ sodium deoxycholate, $0.1 \%$ sodium dodecyl sulfate (SDS), 2 $\mu \mathrm{g} / \mathrm{ml}$ aprotinin, $50 \mu \mathrm{g} / \mathrm{ml}$ phenylme-thylsulfonyl fluoride (PMSF)], then incubated on ice for 30 minutes (min), followed by centrifugation at $12000 \mathrm{rpm}$ for $20 \mathrm{~min}$ at $4^{\circ} \mathrm{C}$. The protein content was determined according to Bradford's method, using bovine serum albumin as a standard. Protein samples were separated by size on $10 \%$ polyacrylamide gel under SDS denaturing conditions, and transferred onto a polyvinylidene difluoride membrane at $12 \mathrm{~V}$ for 2 hours (h). The membrane was blocked in 5\% non-fat milk and incubated with primary antibody against Wnt- 1 , Wnt-2, $\beta$-catenin and C-myc $(1: 300,1: 200,1: 500$ and 1:500 dilution, respectively) overnight at $4^{\circ} \mathrm{C}$. The membranes were then covered with horseradish peroxidaseconjugated secondary antibody for one hour at room temperature. Detection was performed using an enhanced chemiluminescence method. $\beta$-actin was used as a loading control. Positive immunoreactive bands were quantified densitometrically and expressed as ratio of the above mentioned proteins to $\beta$-actin in optical density units.

\section{Immunohistochemistry}

With a cryostat, $6 \mu \mathrm{m}$ sections were cut and then air-dried. Sections were fixed with acetone at $4{ }^{\circ} \mathrm{C}$ for ten minutes. Endogenous peroxidase activity and non-specific staining were blocked with a $3 \%$ hydrogen peroxide-methanol solution and normal goat serum, respectively. Sections were then incubated with primary antibodies overnight at $4^{\circ} \mathrm{C}$ in a humidified chamber. The primary antibodies against Wnt-1 (rabbit polyclonal antibody, Abcam, Cambridgeshire, UK), Wnt-2 (goat polyclonal antibody, R\&D, Minnesota, USA) and C-myc (mouse monoclonal antibody, Santa Cruz, California, USA) were diluted to $1: 50,1: 20$ and 1:50, respectively. The anti- $\beta$-catenin and antiKi-67 antibody (mouse monoclonal antibody, Millipore, Massachusetts, USA) were used at a 1:60 and 1:100 dilution. Secondary antibodies were reacted for $1 \mathrm{~h}$ at room temperature. The sections were then incubated in a streptavidin-avidin complex labeled by horseradish peroxidase and developed using diaminobenzidine. Next, the sections were counterstained with hematoxylin, dehydrated in alcohol and xylene and covered with coverslips. In each immunostaining, PBS was used instead of the primary antibody to serve as a negative control. The expression levels of Wnt-1, Wnt-2, $\beta$-catenin and C-myc in each sample were scored according to the percentage of positive staining cells in at least five high-magnification fields: $0:<1 \%$ positive cells, 1: $1-10 \%$ positive cells, 2 : $10-25 \%$ positive cells, $3: 25-50 \%$ positive tumor cells, 4 : positive cell ratio $>50 \%$. Ki-67 positive cells in each section were counted for label indexing. The percentage of Ki-67-positive cells among $10^{3}$ tumor cells were calculated and statistically compared. The staining was scored in an investigator-blinded fashion.

Table 1: Histologic subtypes and pathologic grades of human brainstem glioma samples

\begin{tabular}{lcc}
\hline Pathological diagnosis & WHO grade & Sample size \\
\hline Ganglioglioma & I & 2 \\
Diffuse astrocytoma & II & II \\
Oligoastrocytoma & II & 3 \\
Anaplastic ependymoma & III & I \\
Anaplastic astrocytoma & III & 3 \\
Glioblastoma & IV & 4 \\
Total & & 24 \\
\hline
\end{tabular}




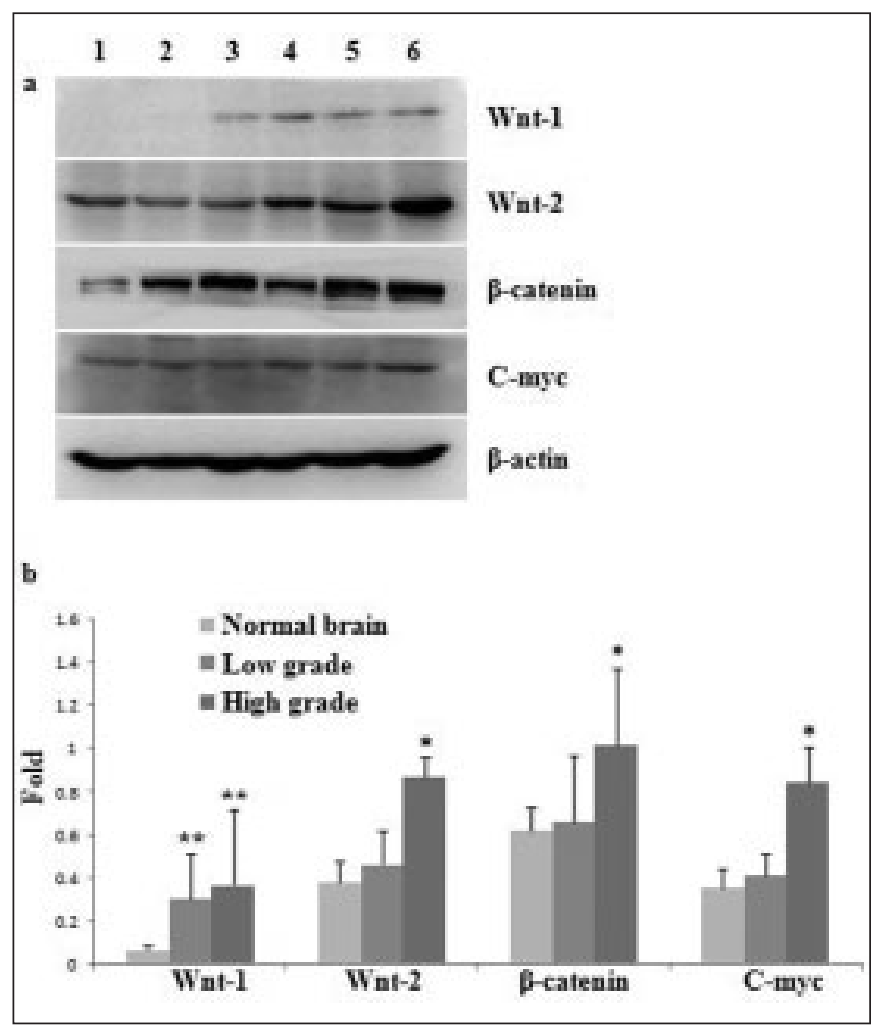

Figure 1: Expressions of Wnt-1, Wnt-2, $\beta$-catenin and C-myc in normal brain tissues and different grades of brainstem gliomas by Western blot. (a) Relative expressions of the above proteins were determined by densitometry. $\beta$-actin served as internal control. Lanes 1-2: normal brain tissues; 3-4: low-grade brainstem gliomas; 5-6: high-grade brainstem gliomas; (b) The expression of Wnt-1 in low- and high-grade brainstem gliomas was higher than that in normal brain tissues. Wnt $2, \beta$-catenin and $C$-myc were overexpressed in high-grade brainstem gliomas as compared with normal brain tissues and low-grade brainstem gliomas. (** compared to normal brain tissues, $P<0.05$; * compared to normal brain tissues and low-grade brainstem gliomas, $P<0.05$ )

\section{Statistical analysis}

All data were expressed as the means \pm standard deviation. Analyses were performed using the statistical software package SPSS 17.0. A one-way analysis of variance (ANOVA) test was used to analyze the expression levels of brainstem gliomas and controls. The differences among clinicopathological variables in different groups were analyzed by the Student t-test or Wilcoxon test. Kaplan-Meier survival curves were generated, and differences in overall survival were compared using the log-rank test. $P<0.05$ was considered significant.

\section{RESULTS}

The expressions of Wnt-1, Wnt-2, $\beta$-catenin and C-myc by Western blot

The expressions of Wnt- 1 , Wnt-2, $\beta$-catenin and C-myc were detected in six cases of normal brain tissues, 16 cases of lowgrade brainstem gliomas, and eight cases of high-grade brainstem gliomas using Western blot analysis. The results of this semi-quantitative analysis show that Wnt- 1 expression in brainstem gliomas was significantly higher than that in normal brain tissues $(P<0.05)$, and its expression did not show any significant difference between high- and low-grade brainstem gliomas $(P>0.05)$; Wnt-2, $\beta$-catenin and $\mathrm{C}$-myc all had different levels of expression in normal brain tissues and brainstem gliomas, and their expressions in high-grade brainstem gliomas were significantly higher than that in low-grade brainstem gliomas and normal brain tissues $(P<0.05$; Figure 1$)$.

\section{Immunohistochemical staining of Wnt-1, Wnt-2, $\beta$-catenin, C-myc and Ki-67}

There was no obvious staining of Wnt-1 in normal brain tissues, but it was expressed in some brainstem gliomas and

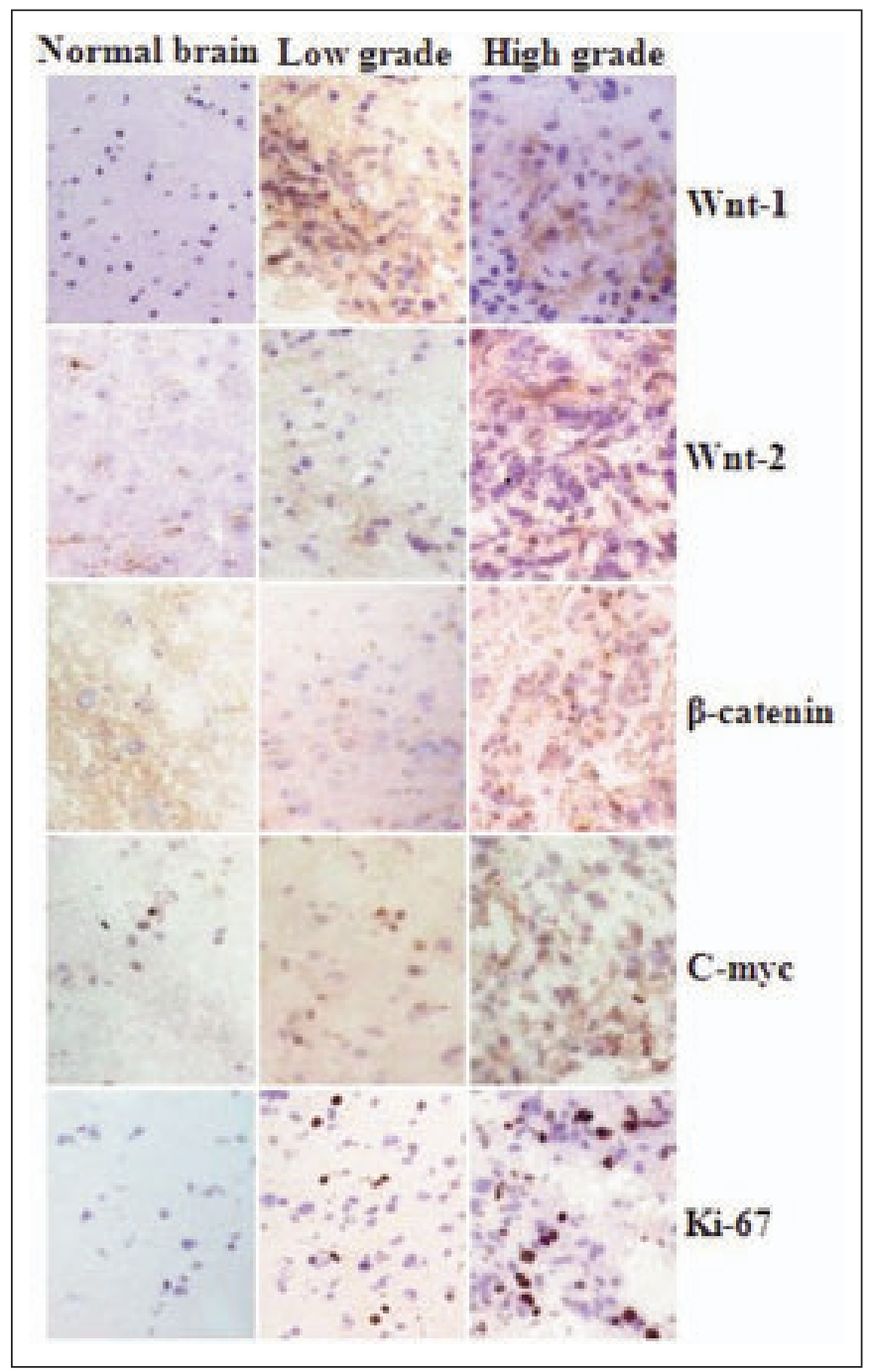

Figure 2: Immunohistochemical staining for Wnt-1, Wnt-2, $\beta$-catenin, $C$ myc and Ki-67 in normal brain tissues and different grades of brainstem gliomas ( $\times 200)$. There was no obvious staining of Wnt-1 in normal brain tissues. Wnt-2, $\beta$-catenin and $C$-myc all had positive staining both in normal brain tissues and brainstem gliomas. Besides, Wnt-2, $\beta$-catenin and $C$-myc expressions were positively correlated with the expression of Ki-67 protein $(r=0.455, r=0.730, r=0.610$, respectively; $P<0.05)$. 
Table 2: Wnt-1, Wnt-2, $\beta$-catenin and C-myc expression levels in human brainstem gliomas with clinicopathological characteristics

\begin{tabular}{|c|c|c|c|c|c|}
\hline Variables & $\mathbf{n}$ & $\begin{array}{c}\text { Score for Wnt-1 } \\
\text { expression }\end{array}$ & $\begin{array}{l}\text { Seore for Wnt-2 } \\
\text { expression }\end{array}$ & $\begin{array}{l}\text { Score for B-catenin } \\
\text { expression }\end{array}$ & $\begin{array}{c}\text { Seore for C-myc } \\
\text { expression }\end{array}$ \\
\hline \multicolumn{6}{|l|}{ Age } \\
\hline$<18$ & 11 & $0.82 \pm 0.87$ & $1.55 \pm 0.82$ & $1.64 \pm 0.92$ & $1.36 \pm 0.67$ \\
\hline$\geq 18$ & 13 & $1.38 \pm 1.04$ & $1.62 \pm 0.77$ & $1.62 \pm 0.77$ & $1.62 \pm 0.51$ \\
\hline \multicolumn{6}{|l|}{ Gender } \\
\hline Male & 14 & $1.07 \pm 1.00$ & $1.57 \pm 0.76$ & $1.64 \pm 0.84$ & $1.43 \pm 0.51$ \\
\hline Female & 10 & $1.20 \pm 1.03$ & $1.60 \pm 0.84$ & $1.60 \pm 0.84$ & $1.60 \pm 0.70$ \\
\hline \multicolumn{6}{|l|}{ Pons } \\
\hline Yes & 9 & $1.33 \pm 1.00$ & $2,00 \pm 0.71$ & $1.67 \pm 0.87$ & $1.78 \pm 0.44$ \\
\hline No & 15 & $1.00 \pm 1.00$ & $1.33 \pm 0.72$ & $1.60 \pm 0.83$ & $1.33 \pm 0.62$ \\
\hline \multicolumn{6}{|l|}{ WHO grade } \\
\hline Low & 16 & $1.06 \pm 0.93$ & $1.38 \pm 0.81^{2}$ & $1.44 \pm 0.89^{2}$ & $1.31 \pm 0.60^{2}$ \\
\hline High & 8 & $1.25 \pm 1.16$ & $2.00 \pm 0.53$ & $2.00 \pm 0.53$ & $1.88 \pm 0.35$ \\
\hline
\end{tabular}

${ }^{\mathrm{a}}$ compared to high-grade brainstem gliomas, $\mathrm{P}<0.05$; WHO=World Health Organization

localized to the cell membrane or juxtamembrane regions of the cytoplasm. Wnt-2 was expressed in both normal brain tissues and brainstem gliomas and had a similar location to Wnt-1 in the cell. $\beta$-catenin was expressed in the cell membrane or juxtamembrane region of the cytoplasm in normal brain tissues, and in the cytoplasm in brainstem gliomas; there was no significant positive nuclear expression. C-myc was localized in the nucleus and expressed in normal brain tissues and brainstem gliomas to different degrees. Ki-67 was not expressed in normal brain tissues, and its expression in brainstem gliomas was significantly correlated with pathological grades and positively correlated with the expressions of Wnt-2, $\beta$-catenin and $\mathrm{C}$-myc $(r=0.455, r=0.730, r=0.610$, respectively; $\mathrm{P}<0.05$; Figure 2$)$.

Association of Wnt-1, Wnt-2, $\beta$-catenin and C-myc immunohistochemical expressions with clinicopathological characteristics

The expressions of Wnt-1, Wnt-2, $\beta$-catenin and C-myc did not show any statistical differences regarding sex, age, and tumor location. The expression of Wnt-1 in high- and low-grade brainstem gliomas did not show significant difference $(P>0.05)$, while the expressions of Wnt-2, $\beta$-catenin and $\mathrm{C}$-myc in highgrade brainstem gliomas were significantly higher than that in low-grade brainstem gliomas $(P<0.05)$; these results were consistent with the Western blot data (Table 2). Furthermore, the results obtained from the survival analysis show that Wnt-1 and $\beta$-catenin expressions were not significantly correlated with the overall survival of brainstem gliomas $(P>0.05)$; however, there was a trend toward increased $\beta$-catenin expression with decreased overall survival; the expressions of Wnt-2 and C-myc were significantly associated with the survival time; the survival of the low-expression group was significantly longer than that of the high-expression group $(P<0.05$; Figure 3$)$.

\section{Discussion}

Gliomas are the most common intracranial malignant tumors, and gliomas in different locations can have different clinical treatment efficacies and prognosis. The overall survival of patients with cerebral hemisphere gliomas can be significantly prolonged through comprehensive treatments such as surgery, radiotherapy and chemotherapy, but the survival of patients with brainstem gliomas usually cannot. Brainstem gliomas account for $1.5-2.5 \%$ of adult brain tumors and $10-20 \%$ of childhood brain tumors. ${ }^{1}$ Although the incidence of brainstem gliomas is not high compared to cerebral hemisphere gliomas, the overall prognosis is poorer, and these tumors severely affect the survival and neurological functions of patients. There are strict indications for brainstem glioma surgery, and a majority of patients are not suitable for operation; ${ }^{6,7}$ radiotherapy usually can only achieve temporary improvement of the symptoms, but cannot prolong survival, and there is currently no precise and effective chemotherapy treatment; ${ }^{8,9}$ thus, there is no mature therapy regimen to significantly improve the clinical prognosis of most brainstem glioma patients. The differences of clinical treatment efficacy between brainstem gliomas and cerebral hemisphere gliomas might be due to the specific location of the brainstem; what's more, there are far more studies concerning the molecular biology and translational medicine of cerebral hemisphere gliomas compared to brainstem gliomas. Under the same pathological nature and treatment methods, the treatment efficacies of cerebral hemisphere gliomas and brainstem gliomas are different, indicating that brainstem gliomas might represent a group of tumors with unique biological features. It is a developing trend to understand tumors including brainstem gliomas from the molecular level to explore the mechanism of their occurrence and development and search for more targeted clinical treatment methods. Unfortunately, due to the limitation of specimen, there are fewer basic studies concerning brainstem 


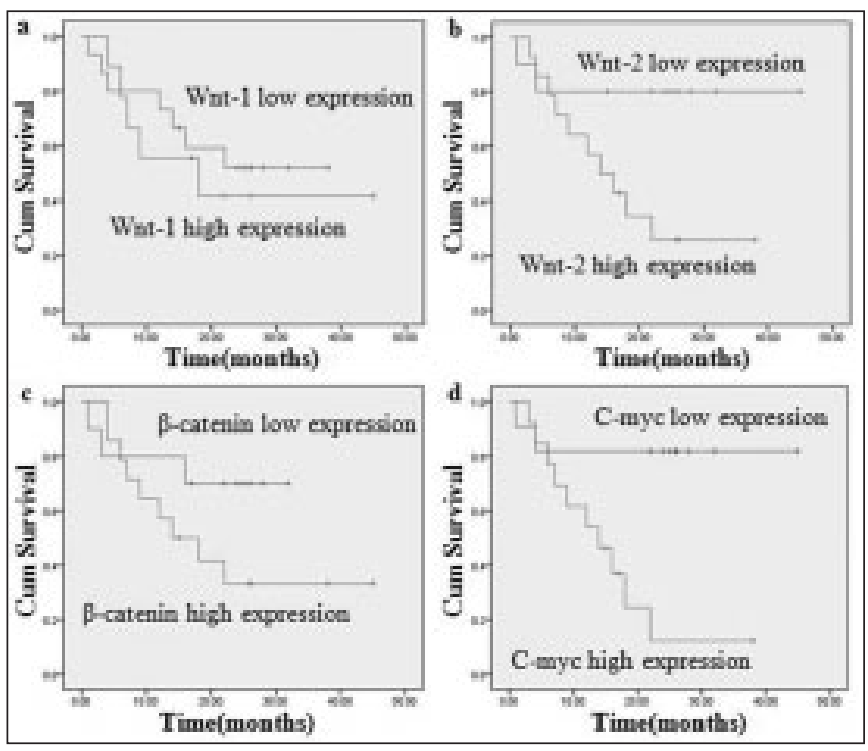

Figure 3: Kaplan-Meier survival curves of the overall survival of brainstem glioma patients with low and high expressions of Wnt-1, Wnt$2, \beta$-catenin and C-myc. (a) There was no significant association between Wnt-1 expression and overall survival of patients $(P>0.05) ;(b)$ The overall survival of patients with low Wnt-2 expression was obviously longer than that with high Wnt-2 expression $(P<0.05)$; (c) There was a trend toward increased $\beta$-catenin expression with shorter survival of patients, but there was no statistical significance $(P>0.05) ;(d)$ Brainstem glioma patients with low $C$-myc expression had a longer survival than that with high $C$-myc expression $(P<0.05)$. (low-expression and high-expression group was scored as 0 or 1; 2, 3 or 4, respectively)

gliomas; thus, the biological features and mechanisms involved in the occurrence and development of brainstem gliomas are still unknown, which limit the progress of the clinical treatment of brainstem gliomas.

Wnt/ $\beta$-catenin signaling pathway plays an important role in the regulation of cell growth and differentiation, embryonic development and the process of tumor occurrence and development. Abnormal activation of $\mathrm{Wnt} / \beta$-catenin pathway is closely associated with the occurrence and development of many human tumors, such as colorectal tumors, lung cancers and liver cancers. ${ }^{10-12}$ In the central nervous system, Wnt/ $\beta$-catenin signaling pathway is closely associated with the occurrence and development of medulloblastoma, which has been well studied. ${ }^{13}$ Additionally, related gene interference and targeted therapy are also being studied. ${ }^{14,15}$ The relationship between $\mathrm{Wnt} / \beta$-catenin pathway and gliomas has been the focus of many biological studies in recent years. ${ }^{16-18}$ At present, it is not clear whether this pathway also plays a role in the occurrence and development of brainstem gliomas and whether it can be used as a new route for targeted therapy against brainstem gliomas.

As the initial proteins of $\mathrm{Wnt} / \beta$-catenin signaling pathway, Wnt proteins are a group of secreted glycoproteins encoded by the Wnt gene. The Wnt gene plays an important role in cell differentiation, polarity, migration and proliferation. There are currently 19 known Wnt protein family members that play important autocrine or paracrine roles. ${ }^{19} \mathrm{Wnt}-1$ and Wnt-2 are the points of interest of present research among the Wnt family members. Our results show that Wnt-1 was not expressed in normal brain tissues but was expressed in some brainstem gliomas, which was consistent with the results of Liu et al, ${ }^{20}$ concerning the expression of gliomas in other locations. Wnt-2 was expressed in both normal brain tissue and brainstem gliomas; its expression in high-grade brainstem gliomas was significantly higher than that in low-grade gliomas and normal brain tissues. Pu et al used immunohistochemistry and reverse transcription polymerase chain reaction (RT-PCR) methods to detect Wnt-2 expression in gliomas and also obtained similar results; in addition, Wnt-2 knockout in human U251 glioma cells also inhibited cell proliferation and invasion and promoted cell apoptosis, indicating that Wnt-2 could be used as a potential target for glioma treatment. ${ }^{21}$

$\beta$-catenin is a multi-functional protein. When Wnt signaling is not activated, $\beta$-catenin interacts with E-cadherin to participate in cell adhesion; after Wnt signaling is activated, $\beta$ catenin phosphorylation and degradation are inhibited, and extra $\beta$-catenin accumulates in the cytoplasm and enters the nucleus to interact with transcription factors and induce the transcription of genes associated with cell proliferation. Therefore, $\beta$-catenin is a major molecule of $\mathrm{Wnt} / \beta$-catenin signaling pathway. Sareddy et $\mathrm{al}^{22}$ studied 32 cases of different grades of supratentorial gliomas and showed that $\beta$-catenin expression in high-grade gliomas was significantly higher than that in low-grade gliomas and normal brain tissues. Our results show that $\beta$-catenin was expressed in the cytoplasm of brainstem gliomas, and the expression increased with increasing pathological grades; the abnormal expression of $\beta$-catenin indicated that the $\mathrm{Wnt} / \beta$-catenin pathway might be activated. The expression of $\mathrm{C}$-myc, as a downstream target gene in this pathway, was higher in brainstem gliomas than in normal brain tissues and its expression increased significantly with the elevation in malignancy among brainstem gliomas. In addition, we detected the expression of the proliferation-related gene, Ki-67, using immunohistochemistry. The results of the correlation analysis show that the expression of Ki-67 was positively correlated with the expressions of Wnt$2, \beta$-catenin and $\mathrm{C}$-myc, further indicating that $\mathrm{Wnt} / \beta$-catenin signaling pathway might be activated to promote the expression of downstream target genes, thus resulting in abnormal cell proliferation and tumor occurrence and development. The specific mechanisms and methods of participation of $\mathrm{Wnt} / \beta$ catenin signaling pathway in the occurrence and development of brainstem gliomas still require further in-depth studies.

At present, there is no evidence regarding the relationship between the $\mathrm{Wnt} / \beta$-catenin signaling pathway and prognosis of brainstem glioma. Our study suggests that Wnt-1 and $\beta$-catenin expressions were not significantly associated with the overall survival of patients. However, there was a trend toward increased $\beta$-catenin expression with shorter survival. The survival of patients with a low-expression level of Wnt-2 and C-myc had significantly longer survival than the high-expression group. Because the subjects were limited in our group, the relationship between Wnt/ $\beta$-catenin signaling pathway and prognosis of brainstem glioma needs to be validated in a larger patient cohort.

In summary, our study show that the expressions of $\mathrm{Wnt} / \beta$ catenin signaling pathway-related components in brainstem gliomas were higher than in normal brain tissues. The 
expressions of Wnt-2, $\beta$-catenin and $\mathrm{C}$-myc were significantly correlated with the pathological grades of tumors. Wnt $/ \beta$-catenin signaling pathway might be activated abnormally and was closely associated with the occurrence and development of brainstem gliomas. In addition, the expressions of Wnt-2, $\beta$ catenin and $\mathrm{C}$-myc were associated with the prognosis of brainstem gliomas and may be used as prognostic marker. Finally, designing small molecular compounds or monoclonal antibodies to target this signaling pathway might be a promising therapeutic strategy for the treatment of brainstem gliomas.

\section{ACKNOWLEDGEMENTS}

This work was supported by the National Natural Science Foundation of China (No. 30772237 and 30900479), Capital Medical Development Foundation (No. 2009-1040) and Beijing Science and Technology New Star Program (No. 2010B121).

\section{REFERENCES}

1. Laigle-Donadey F, Doz F, Delattre JY. Brainstem gliomas in children and adults. Curr Opin Oncol. 2008;20(6):662-7.

2. Rosenthal MA, Ashley DM, Drummond KJ, et al. Brain stem gliomas: patterns of care in Victoria from 1998-2000. J Clin Neurosci. 2008;15(3):237-40.

3. Liu YL, Yang HP, Zhou XD, Gong L, Tang CL, Wang HJ. The hypomethylation agent bisdemethoxycurcumin acts on the WIF1 promoter, inhibits the canonical Wnt pathway and induces apoptosis in human non-small-cell lung cancer. Curr Cancer Drug Targets. 2011;11(9): 1098-110.

4. Yang L, Chen Y, Cui T, et al. Desmoplakin acts as a tumour suppressor by inhibition of the $\mathrm{Wnt} / \beta$-catenin signalling pathway in human lung cancer. Carcinogenesis. 2012;33(10):1863-70.

5. Yeh CT, Rao YK, Ye M, et al. Preclinical evaluation of destruxin B as a novel Wnt signaling target suppressing proliferation and metastasis of colorectal cancer using non-invasive bioluminescence imaging. Toxicol Appl Pharmacol. 2012;261 (1):31-41.

6. Mehta VS, Chandra PS, Singh PK, Garg A, Rath GK. Surgical considerations for 'intrinsic' brainstem gliomas: proposal of a modification inclassification. Neurol India. 2009;57(3):274-81.

7. Mohanty A. Role of surgery in brainstem gliomas. Neurol India. 2009;57(3):231-2.

8. Bartels U, Hawkins C, Vézina G, Kun L, Souweidane M, Bouffet E. Proceedings of the diffuse intrinsic pontine glioma (DIPG) Toronto Think Tank: advancing basic and translational research and cooperation in DIPG. J Neurooncol. 2011;105(1):119-25.
9. Chassot A, Canale S, Varlet P, et al. Radiotherapy with concurrent and adjuvant temozolomide in children with newly diagnosed diffuse intrinsic pontine glioma. J Neurooncol. 2012;106(2): 399-407.

10. Kang YJ, Park HJ, Chung HJ, et al. Wnt//-Catenin signaling mediates the antitumor activity of magnolol in colorectal cancer cells. Mol Pharmacol. 2012;82(2):168-77.

11. Pacheco-Pinedo EC, Durham AC, Stewart KM, et al. Wnt/ $\beta$-catenin signaling accelerates mouse lung tumorigenesis by imposing an embryonic distal progenitor phenotype on lung epithelium. J Clin Invest. 2011;121(5):1935-45.

12. Lachenmayer A, Alsinet C, Savic R, et al. Wnt-pathway activation in two molecular classes of hepatocellular carcinoma and experimental modulation by sorafenib. Clin Cancer Res. 2012; 18(18):4997-5007.

13. Asuthkar S, Gondi CS, Nalla AK, Velpula KK, Gorantla B, Rao JS. Urokinase-type plasminogen activator receptor (uPAR)mediated regulation of $\mathrm{WNT} / \beta$-cateninsignaling is enhanced in irradiated medulloblastoma cells. J Biol Chem. 2012;287(24): 20576-89.

14. Cimmino F, Scoppettuolo MN, Carotenuto M, et al. Norcantharidin impairs medulloblastoma growth by inhibition of $\mathrm{Wnt} / \beta$-catenin signaling. J Neurooncol. 2012;106(1):59-70.

15. Baryawno N, Sveinbjörnsson B, Eksborg S, Chen CS, Kogner P, Johnsen JI. Small-molecule inhibitors of phosphatidylinositol 3kinase/Akt signaling inhibit Wnt/beta-catenin pathway crosstalk and suppress medulloblastoma growth. Cancer Res. 2010;70 (1):266-76.

16. Zhang N, Wei P, Gong A, et al. FoxM1 promotes $\beta$-catenin nuclear localization and controls Wnt target-gene expression and glioma tumorigenesis. Cancer Cell. 2011;20(4):427-42.

17. Rossi M, Magnoni L, Miracco C, et al. $\beta$-catenin and Gli1 are prognostic markers in glioblastoma. Cancer Biol Ther. 2011;11 (8):753-61.

18. Kahlert UD, Maciaczyk D, Doostkam S, et al. Activation of canonical WNT/ $\beta$-catenin signaling enhances in vitro motility of glioblastoma cells by activation of ZEB1 and other activators of epithelial-to-mesenchymal transition. Cancer Lett. 2012;325(1): 42-53.

19. Logan CY, Nusse R. The Wnt signaling pathway in development and disease. Annu Rev Cell Dev Biol. 2004;20:781-810.

20. Liu C, Tu Y, Sun X, et al. Wnt/beta-Catenin pathway in human glioma: expression pattern and clinical/prognostic correlations. Clin Exp Med. 2011;11(2):105-12.

21. Pu P, Zhang Z, Kang C, et al. Downregulation of Wnt2 and betacatenin by siRNA suppresses malignant glioma cell growth. Cancer Gene Ther. 2009;16(4):351-61.

22. Sareddy GR, Panigrahi M, Challa S, Mahadevan A, Babu PP. Activation of $\mathrm{Wnt} / \mathrm{beta}-\mathrm{catenin} / \mathrm{Tcf}$ signaling pathway in human astrocytomas. Neurochem Int. 2009;55(5):307-17. 